\title{
In-Situ Atomic Level Studies of Unusual Phase Transformations in Metal- chalcogenide 2D Crystals
}

Jamie Warner

University of Texas at Austin, Austin, Texas, United States

The family of 2D crystals has expanded rapidly beyond graphene and $\mathrm{hBN}$, with a multitude of crystal structures and stacking types. Understanding the crystal structure response to the loss of atoms and vacancy formation is crucial to the general application of these systems. 2D monolayer crystals of metalchalcogenides have complex atomic structure that is highly sensitive to the elemental concentration in the crystal. This is represented by rich phase diagrams in materials such as $\mathrm{PdSe} 2$. When the light chalcogen atoms are lost from metal-chalcogenide crystals, forming local metal rich areas, several different structural changes occur. In this talk I will cover our latest findings using in-situ heating to cause local S, and Se loss in MoS2, WS2, PdSe2, PtSe2, ReSe2, and discuss the resultant phase transformations. A unique striated channeled lattice occurs in few layered $\mathrm{PdSe} 2$ upon heating, whereby the Se loss leads to $1 \mathrm{D}$ void formation in bilayers. This process eventually leads to large areas of $\mathrm{Pd} 2 \mathrm{Se} 3$ monolayers [1]. This is then compared to PtSe2, which does not form such channeling and instead transforms into PtSe crystals. The grain boundaries and linear defects for these materials will be presented. The beam induced vacancy production will be discussed, along with healing of the vacancies by post-imaging sulfurization. The work will cover the atomic level imaging using annular dark field scanning transmission electron microscopy, combining with in-situ heating stages. I will present our results using 4D STEM for ptychography to generate phase imaging of carbon:metal chalcogenide interfaces in transition metal dichalcogenides, with simultaneous ADF-STEM imaging to enable the discrimination of carbon bonding to metal atoms. Multislice image simulations are used to support the experimental images and models are constructed using density functional theory. The impact of these defects and phase transformations on the device applications will be discussed to bring context to their importance. 


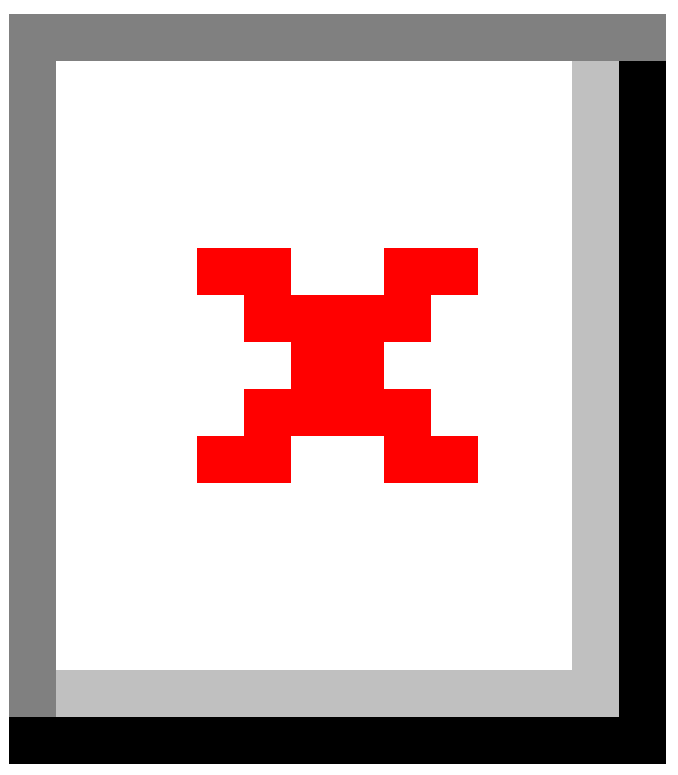

Figure 1. Transformation of few layered PdSe2 into striated Pd2Se3 bilayers

\section{References}

1. H. Ryu, T. Zhu, J. Chen, S. Sinha, V. Shautsova, J. C. Grossman, J. H. Warner, Striated 2D Lattice with Sub-nm Etch Channels by Controlled Thermally Induced Phase Transformations of PdSe2, Advanced Materials, 31, 1904251, (2019) 